\title{
Effect of COVID-19 Pandemic and Lockdown on Children With Gastrointestinal Disorders
}

\author{
Eyad Altamimi a, b, c
}

\section{To the Editor}

\section{Introduction}

Coronavirus disease (COVID-19), caused by the severe acute respiratory syndrome corona virus 2 (SARS-CoV-2), has spread rapidly worldwide [1]. Since first identified on late December 2019 till now, more than two million get infected and more than 150,000 died [2]. This pandemic has overwhelmed healthcare systems and adversely affected an unprecedented number of people [2]. While children have shown resilience and milder symptoms, older adults and those with comorbidities such as diabetes mellitus and cancer seem to manifest more severe forms of this infection and have a higher risk of mortality [3]. The governments of many countries have resorted to the restrictive measures of lockdown and curfew to control the spreading of this disease. The common measures of social distancing, hand hygiene, and reduced hospital visits would apply to all patients [4]. Contact numbers of the treating physician/registered nurse need to be available to reserve in-person consultations for emergencies only [3].

This study reflects on the effect of the COVID-19 pandemic and the restrictive measures on children with known gastrointestinal diseases and has cited examples from Jordan, a country where specific health measures were taken to help those with chronic medical conditions. The repercussions of the enforced lockdown have been discussed under the following sub-headings: Nutritional effect, General considerations of COVID-19 on the gastrointestinal system, and Specific crisis management of children with chronic gastrointestinal disorders, such as chronic constipation, non-alcoholic fatty liver disease (NAFLD), irritable bowel syndrome (IBD), peptic ulcer disease (PUD), eosinophilic esophagitis (EoE), celiac disease, cirrhosis, milk protein allergy, and functional gastrointestinal disorders (FGIDs).

Manuscript submitted April 24, 2020, accepted May 1, 2020

Published online May 8, 2020

aPediatric Department, Faculty of Medicine, Jordan University of Science and Technology, Irbid, Jordan

bediatric Department, King Abdullah University Hospital, Ramtha, Jordan ${ }^{\mathrm{c} C}$ Corresponding Author: Eyad Altamimi, Pediatric Department, Faculty of Medicine, Jordan University of Science and Technology, PO Box 3030, Irbid 22110, Jordan. Email: eyadtamimi@gmail.com

doi: https://doi.org/10.14740/gr1290

\section{Nutritional effect}

COVID-19 is not expected to affect the nutritional status unless the primary presentation is a gastrointestinal disorder that can lead to dehydration. In contrast, patients with significant malnutrition are considered immunodeficient [5], putting them at greater risk of acquiring infections. Theoretically, patients with severe malnutrition are at an increased risk of acquiring COVID-19 and developing a severe disease course. Till date, the effect of the pandemic in areas with a high prevalence of famine and malnutrition has been limited, if not under-reported. Areas affected by famine such as sub-Saharan African countries and those with a high population density, poor sanitation, and restricted opportunities to maintain proper personal hygiene are conducive to the spread of this disease.

On the other side of the spectrum, the risk of significant weight gain during enforced lockdown is apparent. Children's physical activity has become limited while their use of electronic devices for online learning and entertainment has dramatically increased their "screen-time".

Vulnerable children of financially insecure families may be at a risk of malnutrition secondary to limited access to food or changes in eating patterns. Hidden hunger occurs when the quality of food eaten does not meet the micronutrient requirements, and the limited sun exposure during the lockdown may worsen vitamin D deficiency or cause a sub-clinical deficiency to become apparent. Although acute manifestations of vitamin D deficiency are not obvious, its chronic deficiency could affect the immune system. Patients with very low vitamin D levels have a subnormal immune defense. Although there has been no research on the effect of vitamin D levels on COVID-19 susceptibility, extrapolating data on vitamin D supplementation and acquiring respiratory tract infection [6], it may not be unreasonable to supplement children with the maintenance dose of vitamin D during the lockdown.

\section{General considerations of COVID-19 on the gastrointesti- nal system}

For fecal-oral transmission of COVID-19, the SARS-CoV-2 may spread through the fecal-oral route as it replicates actively in human intestinal epithelium and is shed in stools. Active viral shedding could continue up to 7 days after its clearance from the nasopharynx $[7,8]$.

Pediatric patients have a higher risk of transmitting the 
disease due to their poor hand washing techniques. Children should be taught the importance of maintaining personal hygiene after using the washroom through the use of simple easyto-understand language.

Angiotensin-converting enzyme-2 (ACE-2) receptors are abundant at the enterocytes of the ileum and colon. ACE-2 represents the port of entry to the cells, where it replicates and then is shed in the stool of infected patients. This explains the fecal-oral route transmission and the gastrointestinal symptoms of COVID-19 [9]. Gastrointestinal manifestations include diarrhea, vomiting, and abdominal pain, and these manifestations may be the earliest presenting symptoms of COVID-19. Among patients infected, almost one-fourth had gastrointestinal presentations [3].

Direct viral infection, immune-mediated injury, or drug induced liver damage during the treatment of COVID-19 could lead to mild elevations of liver enzymes, although severe liver damage has been reported in some cases [10]. COVID-19 should be suspected in case of a positive contact history even in the absence of respiratory symptoms. To increase the detection rate, it may be worthwhile to perform the liver function tests in these contacts, especially since jaundice is not a common symptom of COVID-19 liver disease.

\section{Specific crisis management of children with chronic gas- trointestinal disorders}

As COVID-19 and SARS-CoV-2 are new, the literature on its impact on children with complex and underlying medical conditions is limited. Children are expected to have same spectrum of disease as adults, but with milder severity $[3,8]$. Inputs on how to maintain the medical management of some specific gastrointestinal disorders during this pandemic are discussed below.

\section{Chronic constipation}

Functional constipation defined as constipation with no organic etiology is the most common cause of constipation worldwide [11]. An increase in the risk factors like low fiber diet, decreased fluid intake and decreased physical activity may be seen during enforced lockdown. Therefore, caregivers of these children are encouraged to continue their medications and maintain adequate hydration to combat this risk. On the other hand, those children who developed constipation due to their refusal to use the school toilets may benefit from the increased time spent at home.

\section{NAFLD}

NAFLD is estimated to affect $3-12 \%$ of children, which will increase up to $80 \%$ of obese children [12]. The main interventions in the management of NAFLD are diet modification and increased physical activity, and these are to be continued as closely as possible. Although the recommended level of activ- ity might be difficult to attain at this time, the food intake and type of foods consumed (healthy, home cooked food vs. fast food) can be regulated while the children stay at home. In addition, the availability of smart devices in almost every home provides multiple options via fitness apps and videos to choose appropriate indoor physical activities.

Another issue in NAFLD patients is the concomitant presence of metabolic disorders like diabetes. COVID-19 is known to be more severe in patients with underlying medical conditions and they need to take extra-precautions regarding social distancing and personal hygiene. Medical follow-up for such patients needs to be delayed if possible to limit their exposure to the hospital environment during this pandemic.

\section{$I B D$}

Children with IBD should continue their medications and be mindful of their self-hygiene. Many of the drugs used to treat IBD suppress the immunity; however, they are unlikely to increase the risk of acquiring the disease [13]. If the patient develops COVID-19, dose-adjustment or discontinuation of steroids may be required [13]. Patients receiving biologic agents for IBD can continue their doses as scheduled. Stopping the treatment for IBD altogether would worsen the patients' clinical condition without significant reduction of the risk of contracting COVID-19. Routine follow-up and endoscopic procedures may need to be delayed [14].

\section{PUD and gastroesophageal reflux disease}

A surge in the incidence of reflux disease could be expected at this stressful time [15]. Gastric acidity represents a major defense mechanism against swallowed pathogens, and till date, there have been no data associating the neutralization of gastric $\mathrm{pH}$ with proton pump inhibitors, with the risk of this infection. Standard measures of protection should be followed.

\section{$E o E$}

There is not enough information to document the effect of this pandemic and lockdown on the patients with EoE. Theoretically, noncompliance to medications may cause recurrence of symptoms and impaction of food in the esophagus requiring urgent upper endoscopy. All patients undergoing such endoscopies should be dealt with as suspected cases of COVID-19. The endoscopy team must comply with the published precautionary instructions and should have adequate training in donning and doffing personal protective equipment [14]. Treatment of EoE with local steroids (budesonide) is unlikely to increase the risk of COVID-19 and can be continued.

\section{Celiac disease}

Data on the relation between celiac disease and COVID-19 is 
almost non-existent. Celiac disease is a chronic autoimmune enteropathy that is managed with a gluten free diet. Patients are encouraged to continue their diet despite limited availability due to food supply chain disruptions. Patients with stable celiac disease are immunocompetent, but non-compliant patients with active celiac disease might have a higher risk than the general population.

In Jordan, the Celiac Disease Care Society freely delivered gluten-free flour to the doors of registered celiac patients with the cooperation of civil defense forces. The society team make themselves available for consultations and nutritional advices.

\section{Chronic liver disease/cirrhosis}

Although the spectrum of disorders leading to cirrhosis and its pathophysiology in the pediatric age group varies widely from that seen in adults, adopting general treatment modalities for cirrhosis is the most appropriate approach at this time. Patients with cirrhosis and those receiving immunosuppressive therapy are theoretically at increased risk of acquiring COVID-19 [16]. Practicing universal precautions in healthcare is mandatory. Till date, no recommendations have been made to reduce the dose of immunosuppressant therapy in these patients.

Regular workup of patients with compensated cirrhosis for varices and risk assessment for hepatocellular carcinoma can be delayed. Those with decompensated liver cirrhosis would require additional vaccination (i.e. pneumococcal and flu vaccines) and prophylaxis for peritonitis and encephalopathy to reduce the likelihood of deterioration that warrants in-hospital treatment [17].

The European Association for the Study of the Liver advises against performing liver biopsies to assess NAFLD or mild elevation of liver enzymes even in patients with a high probability of autoimmune hepatitis and to defer it until an appropriate time [16].

\section{Children maintained on special formulas}

Some children require special formula for the management of metabolic disorders like galactosemia and tyrosinemia, and for treating gastrointestinal disorders like protein intolerance or short bowel syndrome. Alteration or substitution of one formula for another is to be done under the advice of the treating physician or an expert dietitian only. Constant efforts are required to ensure the availability of formula to families in need.

In a country with limited resources like Jordan, lactosefree formula may be considered in older galactosemic patients if soya-based formula becomes unavailable. Patients who are allergic to stable cow's milk protein could use the readily available hydrolyzed formulations if the recommended extensively hydrolyzed formula is in short supply during this crisis.

\section{FGIDs}

Lockdown may affect children with FGID in multiple ways.
The disruption of routine, lack of social contacts, unfamiliar distance learning and worry about their exams may worsen their symptoms [18]. Simultaneously, the home environment in some families may turn hostile with increased stress regarding threats of financial insecurity and domestic abuse, both of which are conducive for the development of FGID in susceptible children.

Parental support, coping exercises, and providing online resources on stress management would help in improving the patients' symptoms. This lockdown has also exposed the need for developing resources in non-English languages.

In Jordan, the civil defense forces have aided patient movement for health interventions (including drug administration) to the hospital and back, and arranged the homedelivery of medications for patients with chronic medical conditions.

In conclusion, COVID-19 represents a serious threat to health worldwide. The restrictive measures adopted by governments to control the pandemic may have adverse effects on societal health. Children seem to be more resilient to the infection and manifest milder symptoms. However, children with chronic gastrointestinal disorders may face specific challenges brought about by the enforced lockdown and these need to be addressed. As we are continuing to learn about this novel viral infection, we have little literature to rely on and data regarding its effects on the pediatric population is miniscule. This paper has tried to address the common pediatric gastrointestinal issues and reflects on some of the specific measures that can be adopted during this pandemic.

\section{Acknowledgments}

The author would like to thank Mr. Omar Altamimi for his help in preparing the manuscript and Editage for English language editing.

\section{Financial Disclosure}

None to declare.

\section{Conflict of Interest}

There is no conflict of interest related to this submission.

\section{Informed Consent}

Not applicable.

\section{Data Availability}

Any inquiries regarding supporting data availability of this study should be directed to the corresponding author. 


\section{References}

1. Wang L, Wang Y, Ye D, Liu Q. Review of the 2019 novel coronavirus (SARS-CoV-2) based on current evidence. Int J Antimicrob Agents. 2020:105948.

2. Coronavirus disease (COVID-19) Pandemic. n.d.). Retrieved April 18, 2020, from https://www.who.int/emergencies/diseases/novel-coronavirus-2019.

3. Guan WJ, Ni ZY, Hu Y, Liang WH, Ou CQ, He JX, Liu L, et al. Clinical characteristics of coronavirus disease 2019 in China. N Engl J Med. 2020;382(18):1708-1720.

4. How to protect yourself \& others, CDC. n.d.). Retrieved from https://www.cdc.gov/coronavirus/2019-ncov/prevent-getting-sick/prevention.html.

5. Bourke CD, Berkley JA, Prendergast AJ. Immune dysfunction as a cause and consequence of malnutrition. Trends Immunol. 2016;37(6):386-398.

6. Martineau AR, Jolliffe DA, Hooper RL, Greenberg L, Aloia JF, Bergman P, Dubnov-Raz G, et al. Vitamin D supplementation to prevent acute respiratory tract infections: systematic review and meta-analysis of individual participant data. BMJ. 2017;356:i6583.

7. Ding Y, He L, Zhang Q, Huang Z, Che X, Hou J, Wang $\mathrm{H}$, et al. Organ distribution of severe acute respiratory syndrome (SARS) associated coronavirus (SARS-CoV) in SARS patients: implications for pathogenesis and virus transmission pathways. J Pathol. 2004;203(2):622-630.

8. Xu Y, Li X, Zhu B, Liang H, Fang C, Gong Y, Guo Q, et al. Characteristics of pediatric SARS-CoV-2 infection and potential evidence for persistent fecal viral shedding. Nat Med. 2020;26(4):502-505.

9. Hindson J. COVID-19: faecal-oral transmission? Nat Rev Gastroenterol Hepatol. 2020;17(5):259.
10. Xu L, Liu J, Lu M, Yang D, Zheng X. Liver injury during highly pathogenic human coronavirus infections. Liver Int. 2020;40(5):998-1004.

11. Vriesman MH, Koppen IJN, Camilleri M, Di Lorenzo C, Benninga MA. Management of functional constipation in children and adults. Nat Rev Gastroenterol Hepatol. 2020;17(1):21-39.

12. Bush H, Golabi P, Younossi ZM. Pediatric Non-Alcoholic Fatty Liver Disease. Children (Basel). 2017;4(6):48.

13. Spencer EA, Ayers T, Sauer C, Zitomersky N, de Zoeten E. NASPGHAN-IBD Committee, COVID-19 general information. NASPGHAN. 2020. https://naspghan.org/ wp-content/uploads/2020/04/NASPGHAN-COVID19and-IBD-Final-V3.pdf.

14. Chiu PWY, Ng SC, Inoue H, Reddy DN, Ling Hu E, Cho JY, Ho LK, et al. Practice of endoscopy during COVID-19 pandemic: position statements of the Asian Pacific Society for Digestive Endoscopy (APSDE-COVID statements). Gut. 2020.

15. Song EM, Jung HK, Jung JM. The association between reflux esophagitis and psychosocial stress. Dig Dis Sci. 2013;58(2):471-477.

16. Boettler T, Newsome PN, Mondelli MU, Maticic M, Cordero E, Cornberg M, Berg T. Care of patients with liver disease during the COVID-19 pandemic: EASL-ESCMID position paper. JHEP Rep. 2020;2(3):100113.

17. American Association of Study Liver Disease. Clinical insights for hepatology and liver transplant providers during the COVID-19 pandemic. 2020. https://www.aasld. org/sites/default/files/2020-04/AASLD-COVID19-ClinicalInsights-4.07.2020-Final.pdf.

18. McOmber MA, Shulman RJ. Pediatric functional gastrointestinal disorders. Nutr Clin Pract. 2008;23(3):268-274. 